\title{
View Planning Approach for Automatic 3D Digitization of Unknown Objects
}

\author{
Souhaiel Khalfaoui, Ralph Seulin, Yohan Fougerolle, and David Fofi \\ Le2i Laboratory, UMR-CNRS 6306, University of Burgundy, \\ 71200 Le Creusot, France \\ \{souhaiel.khalfaoui, ralph.seulin, \\ yohan.fougerolle, david.fofi\} @u-bourgogne.fr \\ http://www.le2i.cnrs.fr
}

\begin{abstract}
This paper addresses the view planning problem for the digitization of $3 \mathrm{D}$ objects without prior knowledge on their shape and presents a novel surface approach for the Next Best View (NBV) computation. The proposed method uses the concept of Mass Vector Chains (MVC) to define the global orientation of the scanned part. All of the viewpoints satisfying an orientation constraint are clustered using the Mean Shift technique to construct a first set of candidates for the NBV. Then, a weight is assigned to each mode according to the elementary orientations of its different descriptors. The NBV is chosen among the modes with the highest weights and which comply with the robotics constraints. Eventually, our method is generic since it is applicable to all kinds of scanners. Experiments applying a digitization cell demonstrate the feasibility and the efficiency of the approach which leads to an intuitive and fast 3D acquisition while moving efficiently the ranging device.
\end{abstract}

Keywords: 3D Digitization, Automation, Automatic Scanning, View Planning, Next Best View, Non-Model-Based Method.

\section{Introduction}

3D models are widely used in many applications such as industrial inspection, computer games, and augmented reality. The 3D model can be generated either artificially by computer assisted designed (CAD) technique or by digitization. The manual 3D acquisition process is very time consuming for human operator since the viewpoints (scanner positions) are selected by a specialized human operator. The quality of the final result strongly depends on the selected viewpoints and thus on the human expertise. Therefore, it is necessary to define a new digitization strategy of objects that minimizes the impact of the human factor. This independence can be obtained by automating the digitization process. When we address the problem of fully automatic 3D digitization of unknown objects, Next-Best-View (NBV) planning is an important approach for the automation procedure. The first goal of view planning is to determine an optimal

A. Fusiello et al. (Eds.): ECCV 2012 Ws/Demos, Part III, LNCS 7585, pp. 496-505, 2012.

(C) Springer-Verlag Berlin Heidelberg 2012 
positioning of the measuring sensor and to complete the digitization using previous views while minimizing the number of acquisitions. The automation of the $3 \mathrm{D}$ acquisition process requires the knowledge of the different components of the measuring system (scanner, positioning system, etc.). Thus, automatic scanning can be decomposed into two phases: the determination of the different poses (position and orientation) and the sensor trajectory generation from previous poses to achieve the next optimal location.

The goal of this work is to automatically generate a complete $3 \mathrm{D}$ model of unknown and complex objects by developing an information-driven approach. Therefore, we introduce a novel NBV strategy based on the evolution of the scanned part orientation. Our method enables fast and complete 3D reconstruction while moving efficiently the scanner. By generating a set of potential views, our technique ensures proper avoidance of unreachable configurations. This paper is organized as follows. In the next section we discuss the related work and briefly define the benchmark methods that will be used to evaluate our approach. In section 3 we introduce our NBV planning method followed by experimental results in section 4 . We conclude in section 5 .

\section{Related Work}

Scott et al. 1 provided a complete survey paper about the view planning problem in the last few decades. The developed non-model-based methods to compute the best views can be classified into two main approaches: volumetric methods and surface methods.

Volumetric approaches are based on the analysis and the knowledge of the workspace state and use voxelization to represent occupied and empty areas. Abidi 2 adapted the concept of entropy in information theory to the problem of volumetric modeling of an unknown object. A utility function is proposed to qualify the amount of acquired data at each stage of the the acquisition process. The local maximum of this function determines the NBV. One advantage is that there is no restriction on the shape, size, and the location of the object. Reed [3] proposed an automatic method in two phases: a modelling phase that constructs a coarse surface mesh from fixed positions, and a planning phase that analyses the model to calculate the next sensor position. Reed determined the visibility volume which is the volume of the space in which a sensor has a direct view on a particular target. Determining the NBV requires the consideration of three constraints (sensor visibility constraint, occlusion constraint, and sensor positioning constraint) which are associated with different volumes. Massios and Fisher [4] introduced a quality criterion in addition to the visibility criterion to improve the overall quality of the acquired data. A voxel representation is used to label voxels as: empty, seen, unseen, or Occlusion Plane. The application of the sensor positioning constraints to the coarse model of the object will determine areas of possible views. This volumetric representation is then projected onto the visibility sphere and the quality criterion is applied to evaluate each view. The direction with the best result is selected. An extension of this algorithm was proposed by Munkelt et al. [5] and is composed of two phases. A first 
initialization phase during which the algorithm maximizes the number of visible voxels respecting a given quality. The second phase maximizes the number of occluded and visible voxels. The stopping criterion of this algorithm is a redundancy rate above a predefined threshold. Connolly [6] used an octree structure to subdivide the space. The determination of the NBV requires an update of this structure that contains four types of node: parent, empty, occupied, and unseen. Two algorithms were proposed: the Planetarium and the Normal algorithm. The Planetarium searches for the area with maximum unseen voxels and detects occlusions caused by the occupied voxels. The Normal algorithm performs a counting of the normals of the unseen voxels that have empty neighbors, resulting on a viewing vector.

The Surface methods use the surface representation of the model to obtain cues about the regions of missing data. Maver and Bajcsy [7] used the model edges to compute the occluded area of each view which was described as a polygon and assumed to be planar. Each edge was classified as active or inactive. A histogram representation of the occluding region and the camera angle was then used to select the next scanning direction. The method proposed by Kok-lim Low [8] takes into account several constraints for the view planning (Acquisition and Quality constraints). From a first acquisition, a partial model of the scene is created. The acquired surfaces are labelled real. The determination of NBV involves assessing the cost of a view for various constraints. The view with the highest score is chosen as the NBV. The view planning is completed if all the views have a score below a predefined threshold. Yuan [9] introduced the concept of Mass Vector Chains (MVC) to characterize closed models. A MVC is defined as the sum of the normal vectors of all surface patches weighted by its projected area in the normal's direction. It was demonstrated that for closed model, the sum of the MVC (Mass Vector Sum (MVS)) is null. The existence of holes in the model causes the MVS to be not null. The opposite of the vector MVS is used as the NBV to complete the model. The algorithm stops only if the object is closed, hence complete. Thus, this method can not be used in the case of complex objects (holes, narrow cavities, etc.). He and Li [10] have added a termination condition using a threshold variation of the surface. The sensor characteristics such as desired resolution, scanning resolution, field of view, and working distance were also included to optimize the scanning process. Loriot [1] established a rapid method composed of two steps. The first phase is based on the MVC approach. The second phase aims to acquire non-digitized areas. This method is very expensive in robot's movements, especially in the first phase of the algorithm as the method of the MVC supports many round trips around the object. Furthermore, this approach does not take into account the distribution of views in the work space since some viewpoints are particularly close to each other. Kriegel et al. [12] determines the candidates for the NBV by calculating points and normals of an estimated quadratic from the different boundaries of the acquired data. This method does not work with large objects or objects that have very sharp angles at the edges. Khalfaoui et al. 13. presented a new method based on the evolution of the bounding box of the object during the acquisition 
process which is based on an arbitrary number of positions computed uniformly using a mid point subdivision. For each step the new data are independent of what was previously acquired. The only information which links two successive acquisitions is the size of the bounding box and not the geometry of the scanned part, which avoids expensive computations on acquired data and leads to a simple and intuitive algorithm.

We reviewed two main approaches: volumetric and surface based methods. For our automation strategy we adopt the surface representation which is more intuitive and simple since it uses directly the characteristics of the acquired data to decide about the NBV. Among the reviewed surface-based methods, we will particularly use MVC [11 and Bounding Box [13] methods for benchmark. The comparison between the different methods will be based on the coverage rate, the number of acquisition, their distribution around the object, and the length of the scanner trajectory.

\section{Proposed Method}

A large number of views improves the accuracy of the resulting model but increases the acquisition time and the $3 \mathrm{D}$ data redundancy. Our goal is to reduce the number of acquisitions while moving the scanner efficiently and to avoid heuristic approaches. The proposed NBV solution is inspired by the human behaviour. When people are given objects they have not seen before, they focus on the edge of the first seen surface, and then they rotate it in order to discover its unseen parts.

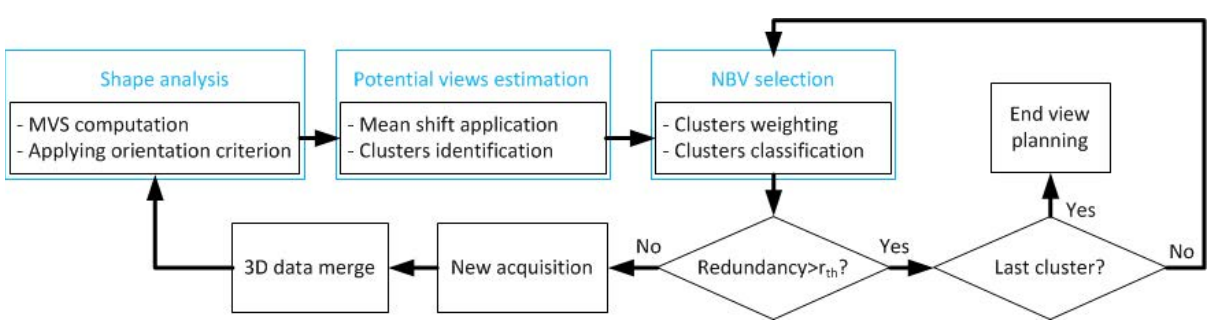

Fig. 1. Flowchart of the proposed method

The developed method is based on the analysis of the orientation of the acquired data at each step of the acquisition process since we assume no prior knowledge about the object shape. The different orientations of the elementary surface patches are classified to determine a set of potential NBVs, so several positioning alternatives of the ranging device, ensuring proper avoidance of robotic singularities and unreachable positions. The next view direction will be chosen between the candidates which satisfy all the constraints of our system. The main stages of the next best view procedure, illustrated in figure 1, are described in detail in the following for the $i^{t h}$ step of the acquisition process. 


\subsection{Shape Analysis}

The next view planning is generated incrementally using the latest acquired data. At each step of the acquisition process, all the previously acquired surfaces are merged to obtain a global surface mesh $S h^{i}$. The resulting model is then analyzed to determine its characteristics. The main orientation of the scanned parts, $M V S^{i}$, is extracted using the MVC technique [9]. The elementary orientation of each surface patch is also calculated and saved in a global orientation vector $\theta^{i}$. One key main idea of our algorithm is to consider only bounded displacements around the last position of the scanner. The $M V S^{i}$ of the rough model changes according to the newly acquired part of the object. Indeed, the addition of new data at the $i^{t h}$ step changes the main orientation of the merged parts at the $(i-1)^{t h}$ step. As shown in figures 2. b and 2. c, the new candidate to the NBV is selected to cover a portion of the data already scanned to guarantee successful registration due to surface overlap.

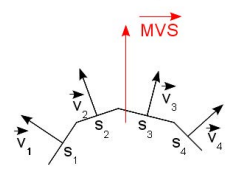

a.

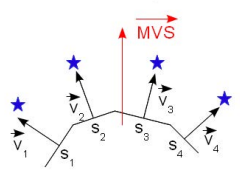

b.

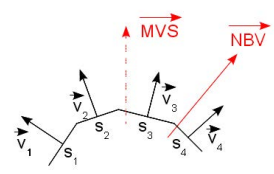

C.

Fig. 2. Next Best View estimation: a) Mass Vector Sum (MVS) determination b) NBV candidates for our method c) Possible NBV

In order to eliminate the view directions close to the $M V S^{i}$, we apply an orientation criterion which allows an initial selection of candidates for the NBV. Under the assumption that the acquired data are not noisy, we consider only the normals with an angle $\theta^{i}>\theta_{t h}^{i}$ close to the $M V S^{i}$ direction. $\theta_{t h}^{i}$ is a threshold angle determined at each iteration as explained in section 4 .

\subsection{Potential Views Estimation}

After the application of the orientation criterion, all the views have the same probability of being selected for the next acquisition. We then apply the Mean Shift technique 14 to cluster all the potential views. The Mean Shift algorithm is an iterative and non-parametric algorithm whose main idea is to treat the data in the $n$-dimensional space as an empirical function of a probability density where dense regions in the space correspond to local maxima or clusters of this distribution. The data associated with the same fixed point are considered members of the same cluster. Each mode is a six-dimensional vector describing the pose of the scanner (Fig. [3). So there is no decorrelation between the position indicator and orientation in determining a potential view direction. Once the set of potential views is computed, the algorithm decide about the optimal next pose of the scanner head. 


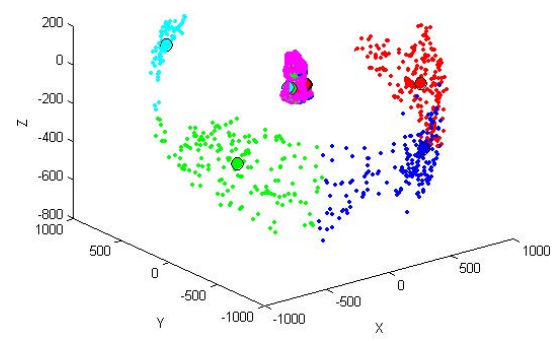

Fig. 3. Determination of four main modes using the Mean Shift technique

\subsection{NBV Selection}

The developed method focuses on the completion of the 3D reconstruction. Since the goal is to explore unseen parts, a weight $w_{k}^{i}$ is assigned to each possible view direction $k$ and is determined among its orientation close to the $M V S^{i}$ as follows:

$$
w_{k}^{i}=\frac{\theta_{k}^{i}}{\theta_{\max }^{i}}
$$

where $\theta_{\text {max }}^{i}$ is the maximum angle value in the orientation vector $\theta^{i}$. Let assume that the $i^{t h}$ clustering step identifies $j$ main clusters $C^{i, l}, l=1: j$. The assigned weight $W^{i, l}$ to the $l^{\text {th }}$ cluster is determined by summing the elementary weights of the different viewpoints belonging to the same set. The weight is calculated as follows:

$$
W^{i, l}=\frac{1}{n^{i, l}} \sum_{k=1}^{n^{i, l}} w_{k}^{i, l}
$$

where $n^{i, l}$ is the number of descriptors forming the set of the cluster $C^{i, l}$. The clusters are sorted according to their weights and the NBV is the one with the highest score. The algorithm switches the NBV from the high weighted cluster to the next one if the redundancy rate is larger than a limit value $r_{t h}$. The stopping criterion is a set of NBVs that ensures a high redundancy between two successive acquisitions.

\section{Experiments}

In our experiments, we used the following values for the parameters of the next best view generation algorithm. For the threshold angle we choose $\theta_{t h}^{i}=\theta_{\text {median }}^{i}$, which is the median value of the orientation vector $\theta^{i}$, since it increases after each acquisition and is less sensitive to noisy data. The amount of new acquired data is set to $3 \%$, which yields to a redundancy threshold of $r_{t h}=97 \%$. 


\subsection{Physical System}

The algorithm was implemented on a robotic cell (see Fig. 4.a) composed of:

- a 6 DoF robotic arm KR16, from KUKA Roboter, with 6 rotational joints.

- a fringes projection scanner, CometV, manufactured by Steinbichler Optotechnik $\mathrm{GmbH}$ with 1.4 Mega Pixels camera and a set of lenses that yields a working volume of $480 \times 360 \times 250 \mathrm{~mm}$ with a working distance of $850 \mathrm{~mm}$. Each acquisition takes 3.5 seconds to be acquired in high quality mode.

- a turntable mounted with its rotational axis vertically and is controlled directly by the robot controller.

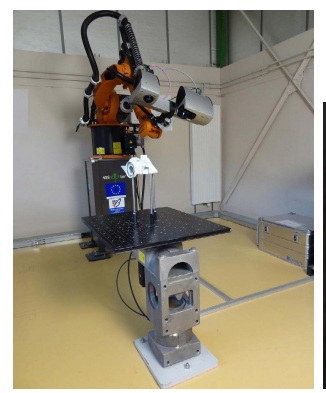

a.

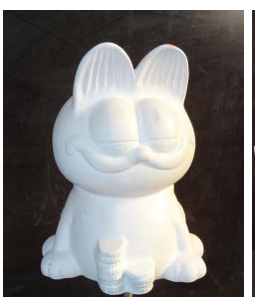

b.

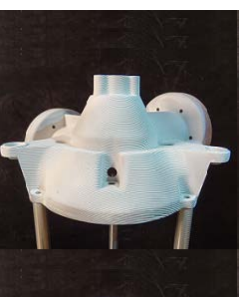

c.

Fig. 4. Experimental setup: a) Robotic cell b) Garfield bust c) Pump-support

\subsection{Results}

We present the results obtained for two objects with different geometries with three benchmark methods: Bounding Box [13], MVC [11, and the proposed method. The first one is Garfield bust, shown in figure $4 \mathrm{~b}$, which has a form similar to a sphere and its size is $192 \times 180 \times 217 \mathrm{~mm}$. The second object is a complex industrial Pum-support (see figure 4.c) which has a medium size of $250 \times 234 \times 145 \mathrm{~mm}$ and presents many concavities and occluded parts.

Table 1 summarizes the different performances of benchmark methods and figures 5 and 6 show respectively the scanner trajectories and the evolution of the completeness rate during the acquisition process. The Bounding Box method is characterized by its simplicity and it leads to a high coverage rate for both models, but needs a large number of acquisitions $(N A=24)$. Although the objects have different geometries, the scanner trajectories are similar. For some acquisitions, the arbitrarily distribution of the viewpoints causes a registration problem since the amount of common information between two successive acquisitions is not sufficient for the pre-alignment. As shown in figure 6 a and 6] b, the evolution of the coverage rate by bounding box for both objects is almost linear because of the uniform distribution of the viewpoints, which yields to an equal amount of new information at each stage of the acquisition process.

The MVC method also ensures a high coverage rate of the objects surfaces. However, this method is very expensive in robot's movements, even for simple 
Table 1. Comparison of the methods: NA and CR and TL and ET are respectively the number of acquisitions, the Coverage Rate, the Trajectory Length and the Execution Time

\begin{tabular}{|c|c|c|c|c|}
\hline \multicolumn{2}{|l|}{ Evaluation grid } & - & + & ++ \\
\hline \multicolumn{2}{|c|}{$\mathrm{Object}^{\text {Method }}$} & MVC & BBox & New method \\
\hline \multirow{4}{*}{ Garfield } & $\overrightarrow{\mathrm{NA}}$ & 28 & 24 & 13 \\
\hline & $\mathrm{CR}(\%)$ & 99.3 & 99.7 & 99.6 \\
\hline & $\mathrm{TL}(\mathrm{m})$ & 22.1 & 14.73 & 8.95 \\
\hline & $\mathrm{ET}(\mathrm{mn})$ & 47.2 & 12.5 & 13.2 \\
\hline \multirow{4}{*}{ Pump-support } & NA & 26 & 24 & 12 \\
\hline & CR (\%) & 97.9 & 98.1 & 98.2 \\
\hline & $\mathrm{TL}(\mathrm{m})$ & 24.1 & 14.5 & 10.32 \\
\hline & $\mathrm{ET}(\mathrm{mn})$ & 45.6 & 12.2 & 13.8 \\
\hline
\end{tabular}

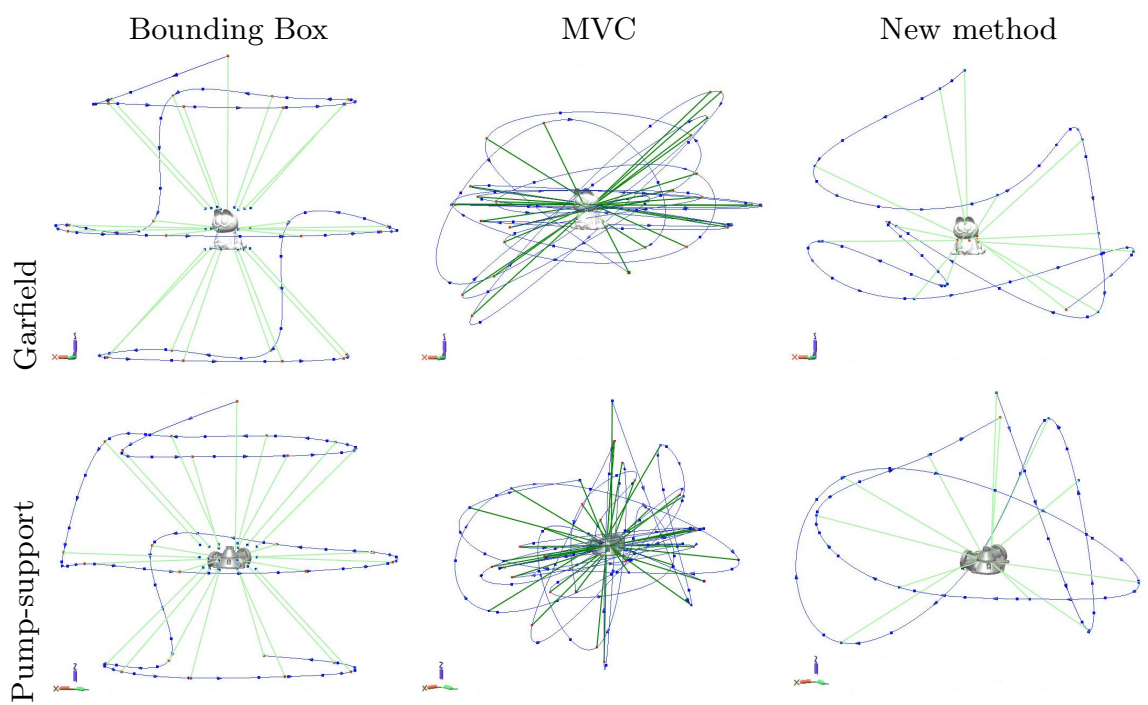

Fig. 5. Trajectories of the scanner during the acquisition process of Garfield and Pumpsupport using the benchmark methods

objects, especially in the first phase of the algorithm as it supports many round trips around the object which yields to many holes in the resulting model. So, several acquisitions are necessary to complete the few amount of missing data. As illustrated in figure 6, b, the MVC performs as a naive approach while scanning complex objects as the pump-support. As shown in figure 5, the scanner trajectory proves that the MVC does not take into account the distribution of views in the work space since some viewpoints are particularly close to each other. Moreover, both methods, Bounding Box and MVC, do not take into account the physical limitations of the acquisition system such as geometric limitations and singularities of a robotic system since they generate only one NBV at each iteration. Our method overcomes these problems while ensuring a high coverage rate for both 
objects. As shown in table 1, the number of acquisition is considerably reduced $(2 \times)$ and the ranging device is moved efficiently around the object along an optimal trajectory (see figure 5) which ensures a progressive and continuous exploration of its different parts. In addition, the proposed technique, contrary to the MVC method, is not sensitive to the initialization step. Indeed, the latter approach constrains the initialization since the first position must be on the sides and not top to the object to overcome collisions with the positioning system. Moreover, our method overcomes the unreachable positions since it computes a set of optimal poses for acquisition. As demonstrated in figure 5, the viewpoints are well distributed around the object which ensures a high coverage of its surface without increasing the $3 \mathrm{D}$ data redundancy. Therefore, this method is less expensive in robot's movements and the scanner trajectory is the well optimised which make the acquisition faster. As illustrated in figures [6 a and 6, b, a complete 3D reconstruction of the object surface is achieved with a minimum number of scans for both test objects. The progressive reconstruction leads to cover a maximum number of holes during the acquisition process. Therefore, it is not necessary to add a second phase, as the MVC method, to fill the resulting holes. The developed method is then very efficient for simple objects as well as complex ones.

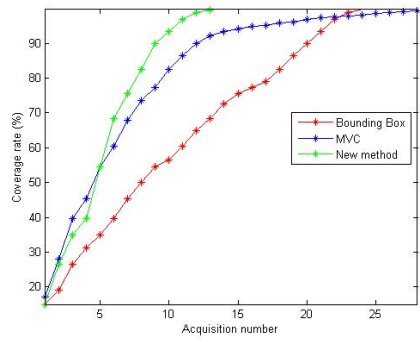

a. Garfield bust

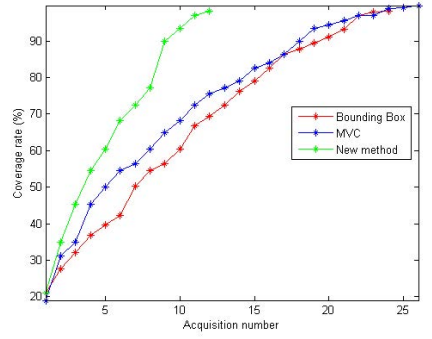

b. Pump-support

Fig. 6. Evolution of the completeness rate (in \%) during the acquisition process

\section{Conclusions and Future Works}

This paper describe an automatic and general surface-based NBV approach. The algorithm does not require any prior knowledge of the object shape. The view planning is based on the analysis of the elementary orientations of the acquired surface patches. A clustering step allows the determination of a set of potential views for acquisition. Using the orientation information, a weight is assigned to each mode and the NBV is the one with the high score. The comparison of our method with a naive method, Bounding Box, and a more advanced technique, the MVC method, confirms the efficiency of our approach while obtaining better results. The method creates the surface of the object by generating viewpoints similar to human intuition and is characterized by its simplicity. This approach permits the determination of a scanner trajectory taking into account the unreachable positions since it computes a set of potential views at each iteration. 
The very good results obtained with our method motivate us to explore another interesting direction for future work which is the digitization of large objects or environments.

Acknowledgments. This work was done within the framework of the LE2I laboratory and financially supported by the Regional Council of Burgundy. The authors would like to thank Mr. Mickaël Provost, Head of Vecteo company (www.vecteo.com), for his technical support and effective collaboration.

\section{References}

1. Scott, W.R., Roth, G., Rivest, J.F.: View planning for automated threedimensional object reconstruction and inspection. ACM Computing Surveys 35, 64-96 (2003)

2. Abidi, B.: Automatic sensor placement. In: SPIE Conf. on Intelligent Robots and Computer Vision XIV, Philadelphia, PA, vol. 2588, pp. 387-398 (1995)

3. Reed, M.K.: Solid Model Acquisition from Range Imagery. PhD thesis, Columbia University (1998)

4. Massios, N.A., Fisher, R.B.: A best next view selection algorithm incorporating a quality criterion. In: Proceedings of British Machine Vision Conference, BMVC (1998)

5. Munkelt, C., Denzler, J., Kuhmstedt, P.: Incorporation of a-priori information in planning the next best view. In: Proceedings of the ISPRS Commission V Symposium 'Image Engineering and Vision Metrology', Aachen, pp. 261-268 (2006)

6. Connolly, C.I.: The determination of next best views. In: Proceedings of the International Conference on Robotics and Automation, St. Louis, Mo, USA, vol. 2, pp. 432-435. IEEE Computer Society (1985)

7. Maver, J., Bajcsy, R.: Occlusions as a guide for planning the next view. IEEE Transactions on Pattern Analysis and Machine Intelligence 15, 417-433 (1993)

8. Low, K.L.: An adaptive hierarchical next-best-view algorithm for 3D reconstruction of indoor scenes. Technical report, Proceedings of 14th Pacific Conference on Computer Graphics and Applications, Pacific Graphics (2006)

9. Yuan, X.: A mechanism of automatic 3D object modeling. IEEE Transactions on Pattern Analysis and Machine Intelligence 17, 307-311 (1995)

10. He, B.W., Li, Y.F.: A next-best-view method with self-termination in active modeling of 3D objects. In: IROS 2006, pp. 5345-5350 (2006)

11. Loriot, B., Seulin, R., Gorria, P.: Non-model based method for an automation of $3 \mathrm{D}$ acquisition and post-processing. Electronics Letters on Computer Vision and Analysis 7, 67-82 (2008)

12. Kriegel, K., Bodenmüller, T., Suppa, M., Hirzinger, G.: A Surface-Based NextBest-view approach for automated 3D model completion of unknown objects. In: Proceedings of the IEEE International Conference on Robotics and Automation (2011)

13. Khalfaoui, S., Aigueperse, A., Seulin, R., Fougerolle, Y., Fofi, D.: Fully automatic $3 \mathrm{D}$ digitization of unknown objects using progressive data bounding box. In: Proceedings of SPIE Electronic Imaging, San Francisco, California, p. 829011 (2012)

14. Comaniciu, D., Meer, P.: Mean shift: A robust approach toward feature space analysis. IEEE Transactions on Pattern Analysis and Machine Intelligence 24, 603$619(2002)$ 